\title{
SITUATION ACTUELLE DES MIGRATIONS INTERNATIONALES : RÉALITÉS ET CONTROVERSES
}

\author{
Alain Morice
}

John Libbey Eurotext | « L'information psychiatrique »

2015/3 Volume 91 | pages 207 à 215

ISSN 0020-0204

Article disponible en ligne à l'adresse :

http://www.cairn.info/revue-l-information-psychiatrique-2015-3-page-207.htm

\section{Pour citer cet article :}

Alain Morice, «Situation actuelle des migrations internationales : réalités et controverses », L'information psychiatrique 2015/3 (Volume 91), p. 207-215. DOI 10.3917/inpsy.9103.0207

Distribution électronique Cairn.info pour John Libbey Eurotext.

(C) John Libbey Eurotext. Tous droits réservés pour tous pays.

La reproduction ou représentation de cet article, notamment par photocopie, n'est autorisée que dans les limites des conditions générales d'utilisation du site ou, le cas échéant, des conditions générales de la licence souscrite par votre établissement. Toute autre reproduction ou représentation, en tout ou partie, sous quelque forme et de quelque manière que ce soit, est interdite sauf accord préalable et écrit de l'éditeur, en dehors des cas prévus par la législation en vigueur en France. Il est précisé que son stockage dans une base de données est également interdit. 


\title{
Situation actuelle des migrations internationales : réalités et controverses
}

\begin{abstract}
Alain Morice
RÉSUMÉ

Ce texte ${ }^{1}$ porte sur la problématique de l'immigration contemporaine envisagée d'un point de vue anthropo-historique. Il envisage d'abord les questions de définition et propose une typologie des divers modèles de mouvements humains. Il rappelle ensuite quelques réalités de ces derniers, parfois malmenées par la classe politique ou par les médias, comme la confusion entre migrants, réfugiés et clandestins, la sous-estimation des migrations entre pays pauvres, ou encore le fantasme d'une menace de l'étranger. Enfin, il examine les politiques migratoires, caractérisées par un décalage entre les discours et les pratiques, la reproduction de rapports de domination et de marchandages entre pays d'origine et d'accueil et par leurs effets délétères sur les candidats à la migration. En conclusion, il pose la question d'une nécessaire discussion sur les conditions d'une liberté de circulation des personnes.
\end{abstract}

Mots clés : définition, migration, migrant, politique, anthropologie, relation internationale, représentation sociale, travail

\section{ABSTRACT}

Current situation of international migration: realities and controversies. This paper $^{1}$ examines the problem of current immigration considered from an anthropological-historical perspective. It first considers the issues of definition and proposes a typology of various models of human migration. It subsequently re-examines a few of these realities sometimes abused by politicians or the media, such as a confusion between migrants, refugees and illegal immigrants, the underestimation of migration between poor countries, or the fantasy of a threat by the foreigner. Finally, it examines migration policy, characterized by a gap between the speeches and practice, the reproduction of the relations of domination and bargaining between countries of origin and destination and their deleterious effects on migration candidates. In conclusion, it raises the question of a needed discussion regarding the conditions for freedom of individual movement.

Key words: definition, migration, migrant, politics, anthropology, international relations, social representation, work

Directeur de recherche au CNRS (laboratoire Urmis, Université Paris-Diderot) et membre du réseau Migreurop (migreurop.org),

36 rue des Plantes 75014 Paris

$<$ morice@univ-paris-diderot.fr $>$

${ }^{1}$ Cet article est issu d'une intervention aux $33^{\text {es }}$ Journées de la Société de 1'Information Psychiatrique (Avignon, $1^{\text {er }}-4$ octobre 2014 ),

sur le thème « Problématiques des migrations aujourd'hui ».

Tirés à part : $A$. Morice 


\section{RESUMEN}

Situación actual de las migraciones internacionales: realidades y controversias. Este documento ${ }^{1}$ versa sobre la problemática de la inmigración contemporánea considerada desde un punto de vista antropo-histórico. Considera primero las cuestiones de definición y propone una tipología de los diferentes modelos de los movimientos humanos. Recuerda a continuación algunas realidades de los últimos, a veces maltratados por la clase política o por los medios de comunicación, como la confusión entre migrantes, refugiados y clandestinos, la subestimación de las migraciones entre países pobres, o aún el fantasma de una amenaza del extranjero. Por fin, examina las políticas migratorias, caracterizadas por un desfase entre los discursos y las prácticas, la reproducción de relaciones de dominación y de mercadeo entre países de origen y de acogida por sus efectos deletéreos sobre los candidatos para la migración. En conclusión, plantea la cuestión de una necesaria discusión sobre las condiciones de una libertad de circulación de las personas.

Palabras claves : definición, migración, migrante, política, antropología, relación internacional, representación social, trabajo

Depuis son irruption sur le terrain électoral comme le signe d'un danger - en France au début des années 1980, puis partout en Europe et dans les pays occidentaux dits $\mathrm{du}$ « Nord »-, la question migratoire fait l'objet d'un ensemble de malentendus, de méconnaissances et de préjugés, marqués par une péjoration lancinante de l'étranger et porteurs d'un climat parfois délétère au sein de populations qui, composites, ne sont pas toujours incitées à bien cohabiter.

Marquée de diverses empreintes, la figure du migrant, cette personne à la fois si convoitée pour ses performances économiques, militaires ou démographiques et si rejetée pour sa supposée inaptitude à s' « assimiler » ou à s' « intégrer » à la société d'accueil poursuit les esprits et les fantasmes [1]. Ces derniers trouvent d'autant plus d'écho que, propulsés par la classe politique et par les médias [2], fort consensuels sur ce point, ils trouvent toujours à être homologués par telle ou telle expérience de tout un chacun. Le " migrant abusif », le " faux demandeur d'asile », le « Rom voleur », l' « Arabe fondamentaliste » etc. : dans les guichets des services de l'État ou dans les quartiers populaires, qui n'a pas sa petite confirmation à apporter à de tels clichés ? Et ne parlons pas des descendants d'immigrés, curieusement nommés « deuxième génération », comme si la condition d'immigré était héréditaire ${ }^{2}$.

Tout cela participe d'un processus d'essentialisation, mode de penser qui est la souche de bien des «-ismes », comme le racisme, le sexisme et le culturalisme. Cette manière de voir autrui a en partage quelques travers cognitifs, parmi lesquels la conjecture, la généralisation, l'exagération, le dénigrement, coiffés ensemble par le complexe de supériorité de qui les énonce [3].

Nous proposons ici un exposé en quatre temps :

\footnotetext{
${ }^{2}$ Voir, à propos d'un enfant de Cubains exilés : « Manuel, l'immigré de deuxième génération, né il y a une trentaine d'années sur le sol américain ». Libération, 19 décembre 2014. Tiens donc : un «immigré » aux États-Unis né aux États-Unis ?
}

- une discussion sur les définitions autour des termes « migrant », ou « immigré » et « migration », qui ne vont pas toujours de soi ;

- le rappel de quelques réalités, qui peuvent heurter le sens commun et que les discours habituels sur la migration tendent à occulter ou déformer ;

- une interprétation critique des politiques migratoires actuelles, d'abord quant à leurs fondements idéologiques et théoriques ; ensuite sur ce qu'elles entraînent dans les relations entre pays donneurs et receveurs ; enfin sur leurs effets, souvent paradoxaux et délétères sur les populations concernées ;

- une conclusion, devant l'accumulation d'impasses, d'injustices et de dénis de droit, sur les solutions alternatives que ces politiques invitent à envisager, y compris dans leurs résultats problématiques, qu'il serait vain d'esquiver.

\section{De quoi parle-t-on ?}

La migration se définit communément comme un mouvement qui implique :

- la traversée d'une frontière extérieure, mais aussi intérieure. Au Brésil, on qualifie de «migrant» soit le saisonnier de la canne à sucre, soit celui qui part de son Sertão natal pour gagner les villes côtières de ce même pays (il en va de même en Russie ou en Chine où, de surcroît, les déplacements sont ou étaient soumis à autorisation). Même après un long temps, « migrant » il restera sa vie entière, et il est bien entendu que les ouvriers du bâtiment comme les «bonnes » sont des « migrant(e)s »- une appellation emplie de contenus dépréciatifs ;

- un projet durable lié à une installation dans le pays d'accueil - là peut surgir une difficulté, liée au souci de ce pays de ne pas pérenniser la présence de l'étranger qu'il a laissé ou fait venir.

Se dessinent là diverses interrogations sémantiques : par exemple, qu'entend-on par « frontière », «projet », « installation »? La définition du « migrant » ou de l' « immigré » cristallise nombre d'entre elles. 


\section{Migrant, immigré}

En France, selon l'Institut national de la statistique et des études économiques (Insee), est « immigré » quiconque, né étranger à l'étranger, réside dans le pays (sous-entendu durablement). Cette définition paraît exclure les touristes, les étudiants, les travailleurs saisonniers, détachés ou en intérim, ainsi que les personnes nées avant l'indépendance dans les anciennes colonies. Pourtant, ces deux ou trois dernières catégories sont le plus souvent incluses dans la catégorisation « migrant ». Le terme est très usité mais mal maîtrisé dans les études sociologiques et politiques. Mais pourquoi qualifier de "migrante » une personne qui est installée depuis des années ? Toute sa vie, le nourrisson qui nous arrivera dans les bras de sa mère sera officiellement un «immigré » ou un « migrant », mais pas le bébé qui sera arrivé à l'état de fœtus : on voit là les limites de ce que véhicule la définition officielle. Quelles sont les arrièrepensées de ce type de qualification, qui suggère soit une légende misérabiliste inspirée des films de Charlie Chaplin, soit plus couramment l'existence d'un marquage à vie, pour ne pas dire d'un stigmate?

La définition du migrant, terme d'emploi commode, n'est pas non plus simple en matière ni du droit des étrangers (qui régit, limite ou favorise leur entrée et leur séjour) ni des droits des étrangers (que le droit leur reconnaît), et encore moins dans l'habituelle confusion entre ces deux registres. Dans un cas, il s'agit, disent les États, d'une prérogative régalienne qu'ils ont dans ce domaine, même s'il lui arrive parfois d'être peu conforme à l'esprit des textes internationaux, tandis que dans l'autre, il s'agit de privilèges inséparables de la personne humaine, indépendamment de toute considération politicienne. On voit là survenir une incohérence, qu'illustrent les deux exemples suivants : d'abord le fait que, dans l'Union européenne (UE), depuis le Traité d'Amsterdam de 1997, l'asile ait été incorporé dans le pilier commun «migration », de sorte qu'un droit fondamental, reconnu par la Convention de Genève de 1951 sur les réfugiés, relève du droit discrétionnaire des États d'examiner ou non la demande de protection qui leur est reconnue. Ce regroupement asilemigration n'est pas innocent et vise à suspecter les réfugiés suspects de faire des requêtes abusives; viennent ensuite toutes les sortes de traitements alternativement discriminatoires ou favorables que réservent des pays comme la Grande-Bretagne aux ressortissants du Commonwealth ou la France aux pays de l'ex-Union française (ou venus des dépendances d'outre-mer, si souvent pourtant perçus et traités en immigrés, quoique citoyens français). Il faut enfin observer que la création en 1995 d'un espace de libre circulation des personnes au sein des pays européens, dit « espace Schengen », amenait nécessairement une hostilité grandissante à l'égard des ressortissants des pays tiers et la cristallisation du symbole dépréciatif associé au mot « migrant ».
Un autre obstacle évoqué plus haut est entretenu conjointement par nombre de politiciens, experts et médias, quand ils parlent des descendants d'immigrés comme étant « d'origine immigrée » : ils le seraient ainsi presque génétiquement et, si l'on fait l'hypothèse hélas ! plausible que beaucoup de gens voient l'immigration comme un mal, alors la condition d'immigré s'assimilerait à une maladie transmissible. Certaines réactions qui suivirent les émeutes de 2005 dans certaines villes françaises, du type « La France, on l'aime ou on la quitte », adressées à des descendants d'étrangers nés dans ce pays et n'en ayant pas connu d'autre, trahissent, par leur absurdité tant politique que scientifique, le climat délétère que, performativement, de tels propos entretiennent.

\section{Migrations et mouvements de personnes}

Comment qualifier les mobilités elles-mêmes, et les personnes qui en sont l'objet ou le sujet (selon la manière différente dont les sociologues et les psychologues les désignent [4]) ? Une classification multiple peut être proposée, dans la tradition weberienne de l'idéaltype, en ayant à l'esprit que chacune de ces distinctions comporte ses contradictions et ses limites. On a ainsi :

- des mouvements définitifs ou provisoires, sans compter le fréquent « provisoire qui dure » (ce qui nous renvoie au mythe des « oiseaux de passage », titre d'un ouvrage de Michael Piore paru en 1979). Sur le thème du provisoire, on peut évoquer celui, devenu en vogue au sein de l'UE, de la « circulation migratoire », dont la fonction, sinon le but, semble être - entre autres - de rendre illégitime l'installation définitive du migrant ;

- des mouvements forcés ou volontaires : là surgit la thématique, très en vogue aussi depuis la convention de Palerme de 2000 sur la « criminalité transnationale organisée » (qui associe sémantiquement l'immigration au crime, voire au terrorisme). Par ailleurs, un des protocoles additionnels est consacré à la traite des personnes et au trafic illicite de migrants, et l'on y comprend que la lutte contre les trafiquants déguise une lutte contre les migrants ;

- semblablement, des déplacements liés à des volontés ou à des contraintes : pour l'anthropologue, l'opposition est ténue car, hormis les affairistes fortunés et capables de nombreux allers et retours, on ne quitte jamais sa terre sans avoir de sérieuses raisons de fuir quelque chose et car, même parmi ceux qu'on nomme « aventuriers », l'insatisfaction, le no future et toutes formes de peurs constituent la force initiale sans laquelle le voyage n'aurait pas été entrepris - l'attraction du pays de destination dit eldorado, qui n'est pas toujours celui qu'on atteindra, tant les frontières sont malaisées à franchir, fera certes le reste ; 
- des mouvements extérieurs ou intérieurs, trop complexes pour être examinés ici en détail. L'exemple suivant illustre cette complexité : au Brésil, nation où la liberté de circuler existe en principe, il peut s'observer une tension entre le droit et les pratiques. Ce pays connaît, à la fin des années 1980, un solde migratoire extérieur quasi nul, voire négatif (ce qui n'est plus le cas actuellement). Par contre, en son sein, les régions riches sollicitent alors les personnes du Nordeste pour immigrer - c'est ainsi que fut édifiée la nouvelle capitale Brasília à la fin des années 1950. Puis on les invite à repartir, mais fréquemment en vain, et grandissent alors les «villes satellites » ou les favelas des grandes périphéries, comme à Rio ! Dans la ville de Bebedouro, (État de São Paulo), spécialisée dans la monoculture intensive des oranges, les autorités appâtent par la radio des Nordestins avant la récolte. Cependant, s'ils arrivent, c'est parfois trop tard, et la municipalité entend bien, jalousement, garder sa prospérité pour ses propres habitants : alors se met en place tout un système para-juridique (intimidations, refus de loger les immigrants et de scolariser leurs enfants, chasse aux sansabri, voire refoulements forcés à la gare routière - autant d'ingrédients d'une xénophobie que nous connaissons trop) destiné à préserver la ville de ses indésirables surnuméraires [5].

Par ailleurs, le mélange problématique entre migration et asile, qui a pour effet généralement d'adosser le statut de réfugié à l'obtention d'une carte de séjour, produit une spirale sans fin entre des tentatives malencontreuses du côté du requérant et les suspicions du côté des agents publics qui lui font face. Il y a là une malsaine contamination de la question des réfugiés par les politiques migratoires, prises globalement. Ces dernières sont porteuses de dangers en matière de droits fondamentaux, lesquels prévoient la possibilité de demander protection dans un autre pays. Aux termes de l'article 13 (alinéa 2) de la Déclaration universelle des droits de l'homme (DUDH) de 1948, « toute personne a le droit de quitter tout pays, y compris le sien ». Cependant, dès les années 1980, les pays de l'UE ont entrepris, systématiquement et avec des moyens associant séduction, coercition et promesses de contreparties, d'empêcher au départ les migrations vers l'espace européen et de faire traiter le «sale boulot » à distance par divers moyens, y compris carcéraux, comme on verra plus loin.

\section{Les migrations, entre réalités et frayeurs}

La diversité des situations historiques rend difficile de se situer à une échelle mondiale atemporelle. Il faut cependant examiner ce que les tendances actuelles du tableau migratoire ont en héritage du passé et ce qu'elles paraissent avoir de nouveau.

\section{II n'est pas de type de migration unique}

Un examen succinct des types de mouvements humains fait apparaître au moins trois modèles migratoires, qui parfois se combinent. On peut distinguer :

- les migrations de colonisation, l'ouverture de fronts pionniers fondés sur la conquête de territoires supposés inoccupés ou occupés par des peuples réputés inférieurs (dont même, commodément, l'appartenance à l'espèce humaine fut jadis considérée comme incertaine, comme lors de la fameuse controverse de Valladolid) : ce sera le cas des deux Amérique et de l'Australie, de l'Afrique australe, de certaines possessions soviétique et chinoise, et plus récemment de la Palestine. Ce type de migration se solde en général par la déportation, le massacre, le confinement ou l'arraisonnement des populations indigènes (avec dans ce cas un statut d'exception qui, ironiquement, n'est pas sans faire penser à celui des immigrés de la catégorie ci-après). Détail important : on demande alors à l'immigrant, une fois sélectionné (parfois brutalement sur des critères eugénistes, ethnico-religieux ou même raciaux comme ce fut le cas dans le centre de tri d'Ellis Island à New York), de rester et de participer activement et durablement à l'édification de la nation : la re-émigration est très mal vue ;

- les migrations contemporaines qui, dans le sillage de la colonisation (non plus alors de peuplement mais d'occupation de comptoirs ou de terres dont les personnes et les productions étaient jugées exploitables), causées par le souci, successivement, de garnir les bataillons en temps de guerre puis de repeupler le pays et faire marcher son économie. Dans le cas de la France, on a vu surgir au $\mathrm{Xx}^{\mathrm{e}}$ siècle des vagues de Polonais, d'Italiens, de Belges, puis de Maghrébins, de Subsahariens, de Portugais et d'Espagnols, dont nombre ont fait souche. Parmi eux, beaucoup furent d'abord acheminés, et ensuite chassés, parfois sans ménagements (à commencer par les travailleurs issus des colonies), comme ce fut le cas lors de la grande crise des années 1930. Quand il ne s'agit pas de mouvements imposés, de nos jours, les demandeurs d'asile, «clandestins » ou candidats à tout autre statut qui atteignent leur but sont mus par la volonté de fuir un ensemble de déficits dans leurs pays : de démocratie, de droits de l'homme et de la femme, de paix civile ou, face à des menaces étrangères, de richesse et de bien-vivre. Cet ensemble de motifs est entremêlé : aussi ne peut-on qu' avoir des doutes sur la pertinence de la distinction répandue entre « vrais » et «faux » réfugiés, respectivement qualifiés de « politiques » et d' « économiques »;

- enfin, les déplacements massifs consécutifs aux guerres, aux persécutions de masse et à diverses catastrophes, qui ont progressivement causé le développement d'une véritable industrie des camps et de l'activité dite humanitaire, dont une des fonctions est de maintenir les personnes au plus près du lieu de la catastrophe et ainsi, pour les pays occidentaux, de se prémunir contre toute « invasion » [6]. Ce phénomène $d$ ' « encampement », pour reprendre un 
anglicisme à la mode, concerne les centres de rétention administrative (CRA) et, dans un contexte plus global, le désir de tenir les personnes déplacées ou non admises à l'écart et à distance : l'actualité nous en fournit, dans le monde, de multiples exemples [7]. La mise à l'écart n'est pas nécessairement de type carcéral : il existe de par le monde une multitude de camps ouverts, comme dans le cas de Sangatte en France entre 1999 et 2002 et des « jungles » qui lui ont succédé, mais aussi de Patras aux marches de la mer Ionienne, ou encore des forêts situées aux alentours des enclaves espagnoles de Ceuta et Melilla en territoire marocain. Leur peuplement répond à un impératif d'invisibilité et, dès lors qu'il n'est plus rempli, les autorités procèdent à l'éradication violente [8] - ils se recréent alors, là ou ailleurs, mais ils se recréent : on ne résorbe rien (comment le pourrait-on ?), mais on déplace sans fin.

\section{Où vont les personnes déplacées ?}

Il est probable que ce dernier type de migration par l'exode massif soit un fait croissant dans le monde - et puisqu'il ne touche statistiquement les territoires des pays riches que très peu, on y tend à ne pas en prendre assez la dimension. Nous le démontre le cas des habitants de Syrie ayant fui la guerre de plus en plus massivement depuis le printemps 2011 vers les pays voisins (Turquie, Jordanie, Liban, Égypte) : presque trois millions à la fin de 2014, contre 3000 parvenus en France ; d'autres exemples peuvent éclairer ce phénomène : celui des centaines de milliers de Rwandais ayant fui vers le Zaïre après les massacres de 1994, ou celui des Subsahariens, tout aussi nombreux, tentant d'échapper à la guerre civile en Libye à partir de 2011 vers l'Égypte, la Tunisie, le Niger et le Tchad (à moins qu'ils n'aient, pour beaucoup - mais cela sera resté une proportion minoritaire -, affronté la mort en mer en tentant de gagner l'Europe).

Héritier d'une vieille tradition dite de « l'asile au plus près » (déjà appliquée après 1948 par l'agence de l'ONU chargée de placer les réfugiés palestiniens), le Haut Commissariat aux réfugiés (UNHCR) a repris, à partir de 2004, la doctrine qui avait fait florès lors de la guerre civile dans l'ex-Yougoslavie des années 1990, nommée avec élégance burden sharing (partage du fardeau), ledit « fardeau » étant les personnes déplacées, dont les pays proches devaient «prendre leur part»: on estime ainsi, aujourd'hui, que sans doute $80 \%$ de cette charge échoit aux pays pauvres et voisins. Tous les thèses y ont été mobilisées par un pays comme la France : ainsi, dans le cas des persécutions subies par les Kosovars en 1999, il fut dit officiellement qu'accueillir des réfugiés de cette région, c'eût été donner l'impression d'accepter « le fait accompli des déportations perpétrées par les Serbes », voire créer une sorte d'appel d'air [9] - l'argument fut utilisé de même autrefois pour les Juifs fuyant l'hitlérisme.

\section{Le syndrome de la menace}

Après avoir été consacrée comme enjeu électoral, dans une surenchère qui n'a cessé de se confirmer, la question migratoire s'est durablement installée comme « problème » - du moins sur la place publique car l'historien Gérard Noiriel fait remonter le phénomène à un siècle plus tôt [10]. On retrouve là cette dimension de pathologie galopante.

À l'origine, ledit « problème » était, dans toute l'UE, l'exclusivité d'un parti d'extrême droite sis en France, quand le coup de tonnerre d'une élection municipale à Dreux en septembre 1983, gagnée par ce parti avec l'appui de la droite classique, révéla que des alliances étaient en train de se faire sur le thème du combat contre l'immigration. Depuis lors, le rouleau compresseur ne cessera de progresser : en France d'abord, où peu à peu l'ensemble des partis parlementaires feront de l'« invasion étrangère » une menace sur l'emploi et sur les bénéfices sociaux des nationaux. Avec l'invocation croissante du péril musulman, elle deviendra un danger que des étrangers réputés inintégrables font peser sur « notre » identité ; dans d'autres pays ensuite, dont la liste n'aura cessé de s'allonger, avec une forte accélération après la chute du rideau de fer, dont les pays européens se mirent à regretter les bienfaits protectionnistes - de fait, sur la route des migrations, en novembre 1989, un rempart était tombé : la France sera rejointe progressivement par de nombreux pays de l'UE, parmi lesquels (liste non exhaustive) l'Autriche, l'Italie, les Pays-Bas, le Danemark, la Grèce, l'Allemagne, la Suède, où fleuriront les partis ouvertement xénophobes, voire racistes. Leurs idées se propageront au sein de l'ensemble de la classe politique, peu à peu prisonnière de telles problématiques.

Dans le vocabulaire de l'UE, on verra se consolider la notion de « risque migratoire » et, parallèlement, un consensus sur la nécessité de combattre l'immigration illégale, incluant les demandeurs d'asile, à tel point que les migrants et réfugiés sont devenus, dans les propos des gouvernements ou dans la presse, eux-mêmes des «illégaux » - appellation choquante quand elle vise des personnes humaines, et provoquant en réaction un slogan des militants dans toutes les langues, tel que nobody is illegal!

Dans des pays d'ancienne immigration comme la France, par une confusion dans l'imaginaire collectif, « immigré » qualifie aussi les enfants d'immigrés, qui ne sont pas eux-mêmes des immigrés. Le thème majeur renvoie alors à un atavisme : « Ces gens-là ne savent (ne veulent) pas s'intégrer ». Tandis qu'Émile Durkheim avait créé le concept d' « intégration » pour traiter de la cohésion d'une société avec elle-même, le terme est devenu une simple injonction, souvent paradoxale : «Ressemblez-nous », paraît-t-on dire à ces descendants français d'étrangers, tout en disant à leur autre oreille que « vous n'êtes pas des gens comme nous ». Il est admis que l'injonction paradoxale peut provoquer la folie [11]. 
Parmi les effets du syndrome de la menace, sur les personnes visées, on peut en distinguer quatre, dont la portée n'est pas assez explorée quant aux désordres psychiques et affectifs qu'ils amènent :

- le poids omniprésent, par contrecoup, de la menace qui pèse sur leurs droits à circuler, à séjourner, à demander protection, à vivre en paix et en famille. Les titres de séjour seront-ils accordés, renouvelés, ôtés ? L'épée de Damoclès plane, en vertu d'une suspicion de tout instant, tandis que l'arbitraire des autorités infantilise les requérants, au point parfois de les dissuader de requérir ;

- la précarité juridique, économique et sociale qui en découle sur tous les domaines vitaux (travail, logement, santé, scolarité), avec les bien connus cercles vicieux, comme l'impossibilité d'ouvrir un compte en banque, voire d'obtenir un numéro de Sécurité sociale, si l'on n'est muni que d'une autorisation provisoire de séjour ou d'un récépissé de la préfecture ;

- les constantes situations de dépréciation, d'humiliation et d'écrasement que cela crée, avec le piège, par un étrange mais fréquent effet performatif, de se faire reprocher ses renoncements ;

- enfin, la tentation de la fraude, de l'infraction, du trafic, des déloyautés comme moyen de survivre en milieu hostile. Si ce thème n'est guère mis en avant dans la littérature scientifique et militante, il mérite pourtant d'être pris en considération, afin de pouvoir comprendre à quelles extrémités les migrants traités comme une menace peuvent être conduits.

Tout cela marche d'un même pied. La rhétorique du « risque migratoire » et de l' « invasion » n'est certes pas nouvelle, et déborde amplement le champ des seuls pays occidentaux. L'histoire contemporaine en fournit divers exemples : ainsi, en Afrique, fut provoqué, par un arrêté de 1969, le départ forcé hors du Ghana d'environ un million de ressortissants de pays limitrophes - le pays en comptait environ huit ; ainsi s'organisa une chasse aux Voltaïques (futurs Burkinabés) en Côte d'Ivoire après la récession caco-caféière des années 1980 ; ainsi, eurent lieu (et continuent) des déportations massives hors d'Afrique du Sud après la révolution de 1995, dépassant parfois le million annuel, de Mozambicains, de Zimbabwéens et d'ouest-Africains, mesures favorisées par des émeutes racistes à répétition dans les grandes villes ; ainsi, les autorités australiennes pratiquent la relégation systématique des demandeurs d'asile ; ainsi, l'État d'Israël lance régulièrement des actions mêlant déportations, enfermement et expulsions de réfugiés venus de zones très risquées pour ces derniers, avec la collaboration parfois meurtrière de l'Égypte. L'invocation de la crise et du danger de se voir confisquer les richesses et son « identité nationale » constituent là, comme en tout lieu, un argument fédérateur.

\section{Les politiques migratoires}

On passera en revue, pour finir, les politiques publiques, puis en conclusion l'alternative de l'ouverture des frontières, en rappelant qu'il s'agit là de " solutions » à un « problème » qui n'en est un que dans l'imaginaire politicien.

\section{Discours et pratiques}

Si l'on s'en tient à l'immigration de travail, les politiques migratoires à l'échelle mondiale ont deux caractères généraux et complémentaires obéissant à des stratégies opportunistes :

- elles obéissent à une dialectique d'attraction-répulsion selon les besoins supposés ou réels, en quantité comme en qualité, des bras étrangers qu'il faudra tour à tour convoquer et congédier. En deçà de la forme juridique jugée adéquate, l'introduction de «clandestins » ne fait pas toujours du tort aux objectifs économiques. C'est ce que l'on peut nommer l'« utilitarisme migratoire » [12];

- elles visent, on l'a vu, à créer les conditions d'un retour forcé (parfois habillé en « volontaire » ou « humanitaire ») vers le pays d'origine, pour éviter tout abcès de fixation des immigrés et de leurs familles. C'est pourquoi l'on a assisté depuis quelques décennies, parallèlement à des formes précaires dites « flexibles » d'emploi, à un développement des introductions d'étrangers par le biais de formules comme le détachement de salariés extérieurs au pays d'accueil, le travail saisonnier et l'intérim organisé du dehors, avec en commun l'obligation pour la personne importée de ne pas élire résidence dans le pays hôte et de retourner dans son pays en fin de contrat: cela sonne la fin de la figure classique, analysée autrefois par le sociologue Abdelmalek Sayad et qui fut chère à Jean-Paul Sartre, du « travailleur immigré ». Ces nouveaux «temporaires » ne sauraient plus être vus ainsi, puisqu'ils ne sont juridiquement que de passage : en somme ils n'existent plus comme immigrés.

Sur de telles problématiques, les pays occidentaux (voire divers pays dits émergents et certains émirats du golfe Persique) semblent assez bien s'accorder; dans le cas de l'UE, la communautarisation des politiques migratoires décidée en 1997 ne concerne que les politiques restrictives ou répressives (voire militaires) contre la migration; pour le reste, les États membres restent chacun jaloux de leurs prérogatives, puisque parfois de forts besoins de main-d'œuvre se font sentir localement, au grand dam des pays voisins, qui redoutent un déferlement induit d'étrangers sur leurs terres. Il y a donc un permanent décalage entre les discours et les faits, et de nombreuses dissensions - les rapports orageux entre l'Italie et la France nous en ont fourni des exemples.

Parallèlement, si l'on revient à toutes les formes de déplacement (migrations de travail mais aussi regroupements familiaux, mouvements de réfugiés et exilés), il est 
utile de s'interroger sur un spectre qui hante les déclarations publiques : celui de l'immigration clandestine, qui est également dans le collimateur de l'UE depuis 1997, avec une forte accélération au début du XXI ${ }^{\mathrm{e}}$ siècle, à la suite des attentats de New York (2001) et de Madrid (2004). Partout, sera consacré un rapprochement entre cette cible et le terrorisme, avec une véritable criminalisation de la première : une fièvre collective, tant obsessionnelle dans les esprits qu'obsidionale dans les faits.

Mais, si l'on s'en tient au cas français, force est de constater au moins deux choses :

- l'une ne se dit guère volontiers, afin de ne pas paraître donner du grain à moudre au parti d'extrême droite, mais tous les gouvernements successifs ont systématiquement régularisé, selon la méthode du « cas par cas », un nombre de « clandestins » à peu près équivalent aux expulsions (soit une trentaine de milliers par an, voire plus $)^{3}$;

- depuis plus de dix ans, les mêmes se fixent pour objectif de limiter l'exercice du droit d'asile, et de prononcer un nombre croisant d' « obligations à quitter le territoire » (OQTF), dont le taux d'effectivité demeure faible. Grosso modo, ces deux choses se compensent.

On est donc fondé à s'interroger sur la fonction de lois et de mesures qui ne remplissent pas les objectifs qu'elles prétendent s'assigner. Cependant, lorsque l'État français, après ses voisins l'Allemagne et la Belgique, a décidé en juillet 1974 de surseoir à toute nouvelle immigration permanente de travail, il n'a pour autant rien stoppé. Peu à peu, on est passé à une logique de flux (les migrants allaient et venaient et se faisaient régulariser au gré de leurs contrats de travail) à une logique de stock : ils affluaient quand même mais, pris dans une nasse, ils restaient. Ce qui constituera une précieuse réserve durable de main-d'œuvre précaire parce que sans droits, pour divers secteurs comme le bâtiment, l'hôtellerie-restauration, l'agriculture, la confection - ce à quoi s'ajouteront ensuite les services à la personne.

L'analyse semble pertinente aussi pour des pays (autrefois d'émigration, maintenant d'immigration) comme l'Italie ou l'Espagne, qui ont fait des régularisations massives à répétition un instrument de leurs politiques migratoires. Mais on note autre chose, qui fut instauré en Suisse dès les années 1930 : en Italie, la loi sanatoria de 2002, dite Bossi-Fini, qui accessoirement instaurait le délit d'immigration clandestine, prévoyait la régularisation d'environ un million de travailleurs à domicile sans papiers - en fait, l'amnistie de leurs employeurs, uniques coupables de délit. De ces derniers, seuls habilités à déposer un dossier, les employés se retrouvaient alors captifs, avec l'embûche de redevenir « illégaux » en cas de rupture de la relation de travail. Pendant la campagne électorale, les initiateurs de la loi avaient prévu un « contrat de séjour », disant que

\footnotetext{
${ }^{3}$ Vincent É. Immigration : les régularisations ont bondi de $50 \%$ en 2013. Le Monde, 10 avril 2014.
}

« l'étranger ne pourra pas séjourner une minute de plus en Italie que la durée de son contrat de travail ». Non conforme aux textes internationaux, cette disposition obligea la loi à prévoir une parade et à astreindre tout étranger changeant d'employeur à aller signer un contrat de séjour à la questura (préfecture) en attestant d'un logement idoine - ces domestiques étaient souvent logés sur leur lieu de travail. Le couple précarité juridique-précarité salariale se révèle très inventif.

\section{L'externalisation}

Quand les diverses formes d'encampement ne paraissaient pas suffisantes, ou quand les inévitables violations des droits de l'homme devenaient trop visibles, les pays ont conçu peu à peu de nouvelles manières de tenir à distance les indésirables.

La chute du mur de Berlin en 1989 sera paradoxalement à l'origine d'une multiplication de murs, aux marges de l'Europe mais aussi à celles des États-Unis, sous diverses formes, matérielles ou virtuelles. On aura là également un marché fructueux et prometteur pour les opérateurs privés, où la dépense publique s'ajoutera à celle des frais, en expansion vertigineuse, des rapatriements forcés et des arraisonnements de navires en haute mer. L'agence européenne Frontex fut créée en 2004 avec un budget de 19 millions d'euros, qui passera en 2011 à 118 millions [13]. Une première incidence est la multiplication des refoulements collectifs et sans examen des situations individuelles vers des destinations où les personnes seront en danger, ainsi triplement en violation des textes internationaux.

Mais le principe de base de l'externalisation vient du constat que ni l'enfermement, ni les retours forcés, ni l'édification de barrières ne produisent les résultats annoncés. A surgi dès lors tout un appareillage idéologique, diplomatique et économique, associant pêle-mêle les notions de coopération, de co-développement et de dialogue « de haut niveau » entre pays riches et pauvres avec celles de la lutte contre les trafics d'êtres humains (dans l'intérêt même, est-il affirmé, de ceux qui en sont présentés comme les victimes) et de la nécessité d'une maîtrise concertée des flux migratoires.

Dans cette perspective, il s'agit de mettre entre les mains des pays tiers le soin d'empêcher à la source ou en chemin les migrations vers les pays nantis. C'est ainsi que fut forgée la notion de "lutte contre l'émigration illégale », dans la droite ligne de ce qui prévalait à Berlin-Est, où tout « fuyard » pouvait se faire fusiller du haut des miradors. En 2002 et 2003, sous les injonctions de l'UE, le Maroc et la Tunisie firent voter des lois contre l'«émigration illégale », et ainsi il advint à l'automne 2005 que l'armée marocaine tua plus de dix personnes qui tentaient de franchir les grillages des enclaves espagnoles et en déporta d'autres dans le désert [14]. Certes révoltant, l'événement eut l'intérêt de montrer les bénéfices attendus d'une mise en 
scène : soucieux de faire savoir qu'il saurait se comporter en bon gendarme de l'Europe et qu'il méritait les sommes que cette dernière lui avait promises, le Maroc prit en effet soin de faire venir des reporters européens pour filmer le mauvais sort réservé aux personnes capturées.

L'expression "émigration illégale » a fait florès, non seulement dans la presse mais aussi chez certains politiciens, comme en 2006 le futur président de la république française, qui salua lors d'une conférence internationale qu'il présidait au nom de l'UE « les efforts des pays de la rive sud de la Méditerranée pour contenir l'émigration illégale vers l'Europe ». Les États-Unis et le Canada ne font pas autrement avec le Mexique face à ses voisins d'Amérique centrale.

Ces orientations sont source de marchandages entre dirigeants, eux-mêmes à la base d'une consolidation des inégalités entre pays et donc, par une perverse circularité, cause de migration accrue. Les tractations sont de tous ordres : retenons seulement d'abord la soumission des accords commerciaux et de l' « aide au développement » (bien souvent un autre mot pour la corruption des dirigeants) à la bonne volonté des pays tiers en la matière, comme ce fut par exemple prévu par les accords de Cotonou signés en 2000 entre l'UE et quatre-vingts pays du groupe Afrique-caraïbes-pacifique (ACP). Plus généralement, moyennant compensations, il s'agit d'obtenir que les pays tiers empêchent les départs ou les transits par leur territoire : le Maroc, la Turquie et, grandement, la Libye ont été des terrains d'expérimentation; ensuite, dans le prolongement de cette doctrine unilatérale, viennent les « accords de réadmission », dont le mécanisme est ambivalent : s'il est nécessaire en théorie que les pays reconnaissent leurs ressortissants afin qu'il puisse être procédé à leur expulsion, les pays d'origine n'y trouvent guère d'intérêt en fait, sauf à utiliser ces émigrants comme une monnaie d'échange, et donc à reporter éternellement la signature de tels accords.

L'externalisation peut enfin provoquer la création de camps à distance. Ainsi, depuis 2001, avec la Pacific Solution, l'Australie s'est-elle fait une spécialité des renvois forcés vers des contrées où elle a passé accord : outre l'île Christmas (qui est sa possession, mais à une distance dissuasive de plus de $2600 \mathrm{~km}$ de Darwin ou Perth), site d'une sorte de bagne souvent comparé à celui de Guantanamo, plusieurs lieux de déportation forcée firent l'objet de conventions, moyennant finances, comme avec Nauru, la Papouasie et aujourd'hui le Cambodge, disposé à acheter les réfugiés en vue de les enfermer ou (hypothétiquement) de les retourner dans leurs pays. Il s'agit de camps de concentration, qui échappent totalement au contrôle international, comme ce fut le cas de ceux créés en Libye dans les années 2000 à l'initiative de l'Italie et de l'Allemagne, ou à la frontière entre le Zimbabwe et l'Afrique du Sud, souvent gérés par l'Organisation internationale pour les migrations (OIM), très présente dans de telles opérations [15]. Déjà en 2003, la Grande-Bretagne avait proposé de créer des pro- cessing centers pour les demandeurs d'asile dans des pays situés hors des frontières de l'UE et peu sûrs en matière de respect des droits humains.

\section{Quels effets sur les personnes?}

Rappelons pour mémoire la fragilisation des individus et la déstabilisation constante qui pèse sur eux, toutes conditions confondues : demandeurs de visa, d'asile, d'une autorisation provisoire de séjour (APS), de renouvellement d'un titre, de regroupement familial, etc. Le terme « demandeurs » fédère bien des désordres psychiques, par le fait même qu'il les met en permanence dans un étau vis-àvis des autorités (mais aussi des associations censées les secourir, sinon de leur voisinage) entre la suspicion et une dépendance infantilisante. Les enquêtes font ressortir la dimension d'un tel phénomène, de même que la manière dont il est si souvent ignoré des services de contrôle ou d'accueil, indiquant que ces personnes ne sont pas vues comme des personnes. La constitution structurelle d'une classe de sans-papiers, aisément corvéables mais sans cesse sur le qui-vive, y contribue.

Trois conséquences des politiques migratoires répressives parmi d'autres sur les migrants, réfugiés et exilés, sont insuffisamment présentes dans les analyses.

D'abord, à la lutte contre un « risque migratoire » imaginaire, fait pendant une prise de risque, réelle cellelà, et croissante, par les candidats au voyage : plus les frontières se ferment, et plus les migrants empruntent des voies dangereuses. L'OIM a recensé 23000 personnes mortes en Méditerranée, dans le désert ou ailleurs, de 2000 à l'automne 2014 mais, pour la seule période janvierseptembre 2014, le bilan s'élevait déjà à 3100 noyades - ces chiffres, qui par ailleurs manquent de clarté, ne peuvent être que sous-estimés et l'augmentation est exponentielle. Les drames épouvantables qui ponctuent les voyages terrestres, comme la traversée du Sahara ou celle du Mexique, restent mal connus et mal évalués.

Ensuite, indissociable de cela, on assiste à un renforcement et à une concentration du rôle des intermédiaires. Souvent désignés à la vindicte publique comme «passeurs », quoiqu'ils ne constituent pas une classe homogène, ils ne sont que le produit d'une politique de fermeture aux migrations : ils remplissent une fonction et n'existeraient pas sans cette politique. Par ailleurs, les pratiques criminelles associées aux voyages, en forte expansion, sont à mettre en rapport avec les décisions xénophobes qui en sont le terreau.

Enfin, encore dans le même sens d'une fragilisation accrue des voyages, on voit partout une floraison de prédateurs, mêlés auxdits intermédiaires ou non, qui pillent les épargnes des personnes, les renvoient souvent à la case départ, et obligent à leur tour les migrants à se livrer à des trafics ou à accepter de se faire exploiter [16], les femmes à se prostituer, tout cela en vue de reconstituer le cash flow 
qui leur fut dérobé et qui leur permettra de reprendre la route [17]. Fréquemment, la connivence entre la police ou l'armée et les voyous pilleurs de migrants est de notoriété publique [18].

\section{Conclusion : ouvrir les frontières ?}

Pour conclure, l'ensemble de ces effets sur les populations prises pour cible, associé à l'inefficacité avérée des politiques migratoires répressives et à leur coût devenu pharamineux, voilà qui appelle une double interrogation : - s'agit-il d' « humaniser » le contrôle des frontières, les modalités d'accueil comme de refoulement? La mécanique décrite ici et ses logiques ne portent guère à y croire, non plus que le consensus qui s'est peu à peu consolidé entre tous les partis ;

- ne s'agit-il pas plutôt, positivisme à part, de se demander si le temps n'est pas venu d'une discussion organisée sur la liberté de circulation et d'installation des personnes dans le monde ? Il y a vingt ans, cela appelait de peu aimables imputations d'utopie, parfois insultantes, au nom de la sacro-sainte notion d'État-Nation. Il se trouve que désormais, singulièrement depuis la publication d'un rapport de l'Organisation des nations unies pour l'éducation et la culture (Unesco), les séminaires et rencontres se multiplient [19].

Il s'agit bien sûr de savoir à quel prix cette libre circulation : dans tous les pays ensemble sans doute, mais aussi par un combat simultané pour le maintien d'un droit du travail et contre le racisme, afin d'éviter que cette liberté ne soit le prétexte d'un dumping social et d'un écrasement discriminatoire des étrangers. Le défi est là, plus à portée de main qu'autrefois.

Liens d'intérêts: l'auteur déclare ne pas avoir de lien d'intérêt en rapport avec cet article.

\section{Références}

1. Collectif. Figures de l'étranger, quelles représentations pour quelles politiques ? Paris : Gisti, 2013. Coll. « Penser l'immigration autrement ».

2. Bourdieu P. L'opinion publique n'existe pas. Les temps modernes $1973 ; 318$ : 1292-309.
3. Guillaumin C. L'Idéologie raciste, genèse et langage actuel. Paris/La Haye:Mouton, 1972 [rééd. : Gallimard, 2002].

4. Despret V. Hans : Le cheval qui savait compter. Paris : Le Seuil/Les empêcheurs de penser en rond, 2004.

5. Bailby E. Quelque part au Brésil : Bebedouro, où les oranges ont un parfum de prospérité. Le Monde Diplomatique 1992: 16-7.

6. Agier M (dir.). Un monde de camps. Paris : La Découverte, 2014.

7. Agier M. L'encampement comme nouvel espace politique. Vacarme $2008 ; 44: 80$.

9. Clochard O (dir.). Atlas des migrants en Europe. Géographie critique des politiques migratoires. Paris : Armand Colin, 2012.

9. Rodier C. Révolutions arabes : des héros, mais de loin. Plein Droit $2011 ; 90: 3-5$.

10. Noiriel G. L'immigration : naissance d'un « problème » (1881-1883). In : Hmed C, Laurens S (dir.). L'invention de l'immigration. Marseille : Agone 2008, 40 : $15-40$.

11. Bateson G. Steps to an Ecology of Mind (Morale and National Character). Chicago : The University of Chicago Press, 2000.

12. Morice A. "Choisis, contrôlés, placés" - renouveau de l'utilitarisme migratoire. Vacarme 2001 ; 14 : 56-60.

13. Rodier C. Xénophobie business. Paris : La Découverte, 2012.

14. Blanchard E, Wender AS. (coord.) Le Livre noir de Ceuta et Melilla. Paris : Migreurop, 2006.

15. Pécoud A. Que sait-on de l'Organisation internationale pour les migrations ? In : Clochard O (dir.). Atlas des migrants en Europe. Géographie critique des politiques migratoires. Paris : Armand Colin-Migreurop, 2012, p. 76-77.

16. Gatti FB. Sur la route des clandestins. Paris : Éditions Liana Levi, 2008 [«Bilal : il mio viaggio da infiltrato nel mercato dei nuovi schiavi », 2007].

17. Brachet J. Irrégularité et clandestinité de l'immigration au Maghreb. Cas de l'Algérie et de la Libye. In : Ali Bensaâd A (dir.) Le Maghreb à l'épreuve des migrations subsahariennes. Immigration sur émigration. Paris : Karthala, 2009, p. 109-35.

18. Morice A. Être Mozambicain dans le Mpumalanga. Lusotopie 2009; XVI(1) : 99-112.

19. Pécoud A, de Guchteneire P. Migrations sans frontières, essais sur la libre circulation des personnes. Paris : Unesco, 2009. 